\title{
Etude exploratoire auprès des enseignes de la grande distribution : Dans Quelle Mesure les Bénéfices des Programmes de Fidélisation Peuvent-Ils Conduire à Une Fidélité à l'enseigne ?
}

\author{
Amina LAGZOULI* Pr. Redouane BARZI \\ ENCG Kenitra, Université Ibn Tofail, Morocco
}

\begin{abstract}
Résumé
Cet article a pour objectif d'analyser l'influence des bénéfices (utilitaires, hédoniques et symboliques) des programmes de fidélité sur la fidélité à l'enseigne. Cette analyse, repose sur une exploration théorique des travaux portant sur la fidélité des clients. Aussi sur une étude qualitative exploratoire présentée par un guide d'entretien destiné aux responsables des enseignes dans la grande distribution. L'étude auprès de ces responsables nous a permis d'appréhender la réalité du terrain et d'affiner notre problématique étudiée pour conduire une recherche quantitative plus précise. Les résultats de cette étude nous ont permis de préciser les conséquences de la fidélité suivants : bouche à oreille positif, intention de fidélité, part du portefeuille client, résistance à la contre persuasion et l'achat répété.
\end{abstract}

Mots-clés : programmes de fidélité, fidélité à l'enseigne, conséquences de la fidélité.

DOI: $10.7176 / \mathrm{JMCR} / 70-05$

Publication date:August $31^{\text {st }} 2020$

\section{Introduction}

Le secteur de la grande distribution est en évolution rapide, caractérisé par une concurrence acharnée dans la détermination des parts de marché. Ceci est souligné par la transformation de l'environnement, l'existence des clients opportunistes et exigeants, le positionnement des enseignes classiques sur de nouvelles valeurs (du gigantisme des années 80 et 90 aux supermarchés de proximité), l'éclosion de la technique du drive, la préférence des produits bio, l'avènement du marketing digital et la révolution dans les technologies du commerce électronique. Dans un tel environnement, les consommateurs ont un large choix des enseignes et sont en mesure de sélectionner ceux qui offrent le plus de valeur. Dans ces conditions, le maintien de la fidélité des clients est essentiel et présente de nombreux défis pour les enseignes (Omar, Ramly, Alam et Nazri 2015). L'une des méthodes employées par les responsables pour acquérir la suprématie est de différencier leurs offres par rapport à celles des concurrents dans les marchés cibles. Pour cette raison, les outils de marketing relationnel sont opérationnels afin d'améliorer la compréhension et l'amélioration des relations avec les clients (Bojei, Julian, Wel et Ahmed 2013). L'objectif principal d'une stratégie de marketing relationnel est d'améliorer la fidélité des clients par le développement de relations (Aurier et de Lanauze 2012). L'une des techniques populaires dans cette approche est la mise en œuvre d'un programme de fidélisation (Kreis et Mafael 2014 ; Meyer-Waarden, Benavent et Castéran 2013). Ce programme est un moyen très efficace pour valoriser les meilleurs clients, maintenir les ventes et obtenir des informations sur les préférences des adhérents.

Les programmes de fidélisation se distinguent des autres formes de promotion par leur nature à long terme et mettent l'accent sur la préservation de la fidélisation de la clientèle et l'intensification de la fréquence d'achat (Lacey \& Sneath 2006). Henderson, Berk et Palmatier, (2011); Meyer-Waarden, (2007 et 2012) ; Sharp et Sharp, (1997) ont défini les programmes de fidélité en tant qu' un système d'incitation qui comprend une variété d'initiatives marketing à long terme (cartes de fidélité, cadeaux, services additionnels à plusieurs niveaux, relations personnalisées, etc.) ainsi que d'autres méthodes qui influencent positivement les attitudes et les comportements de fidélité à l'égard du programme, de la marque et de l'entreprise.

Notre étude se concentre sur le marché de la grande distribution au Maroc. Ce marché présente un intérêt particulier, en raison de la nature concurrentielle au niveau de l'industrie du commerce de détail, et des difficultés que rencontrent les magasins pour se différencier au sein de ce marché. En effet, le gouvernement fait de la croissance de ce secteur une priorité, en proposant un programme national baptisé Rawaj Vision 2020, ce programme est destiné pour le développement du secteur à travers des initiatives de modernisation et se donne pour objectif de faire passer la contribution du secteur du commerce de détail au PIB à $15 \%$ et de créer 450000 emplois supplémentaires et une croissance annuel de $8 \%{ }^{1}$ à l'horizon $2020^{2}$ (Chambre de commerce d'industrie et de services CCISDT).

Dans le Royaume, le nombre de programme de fidélité continue d'augmenter, avec plusieurs types de cartes de fidélité qui opèrent à l'échelle nationale. Une grande partie de cette croissance a eu lieu au cours des cinq à dix

\footnotetext{
${ }^{1}$ Chambre de commerce d'industrie et de services Rabat, Salé et Kenitra : https:/www.rabatinvest.ma/articles/le-commerce

${ }^{2}$ Chambre de commerce d'industrie et de services CCISDT : https://ccisdt.ma/fr/programme-rawaj-vision-2020. Consulte le 02 octobre 2019.
} 
années précédentes. Selon les chiffres publiés ${ }^{1}$ par le Centre Monétaire Interbancaire, la plateforme de fidélité ${ }^{2}$ compte plus de 50000 utilisateurs, ce programme a permis une augmentation dans le volume des opérations de paiement des cartes marocaines avec un taux de $22,5 \%$ dans les points de vente et $82,1 \%$ via Internet. Ces chiffres témoignent aussi que le nombre des transactions par carte bancaire a évolué avec un taux de plus de $96 \%$ et le montant de paiement de plus de $116 \%$ par rapport à la même période de l'année dernière. De même, en 2019 l'enseigne Aswak Assalam est arrivée à plus d'un million abonnés à la carte de fidélité dont 50\% sont toujours actifs depuis 2009, cette amélioration a été confirmée par l'enseigne dans un communiqué du groupe Chaabi «Le panier moyen des clients détenteurs de la carte de fidélité s'est vu tripler en moins de cinq ans et la fréquence de visite des hypermarchés est passée de 2 à 5 fois par mois» ${ }^{3}$.

Par ailleurs, les programmes de fidélité des enseignes marocaines se trouvent manquants en les comparants à l'offre internationale, en termes d'exécution, de communication et de différenciation de ses offres. A titre d'exemple les enseignes en États-Unis consacrent 48 milliards de dollars par an dans les points (miles) (Melnyk et Bijmolt, 2015), en France, le coût du programme de fidélité est compris entre 80 à 120 millions d'euros selon le nombre des adhérents et transactions (Lars Meyer-Waarden 2016). En plus 77\% des consommateurs français participent a au moins un programme de fidélité (Nielsen ${ }^{4}$ 2016). Bien qu'un certain nombre des enseignes éminents au Maroc offrent des cartes de fidélité, d'autres ne les ont pas encore adopté ou n'ont pas l'intention de les adopter, pour cette raison, notre étude présentera aux responsables de gestion des programmes de fidélité, des informations et des explications sur la fidélité à l'enseigne et sur la question de savoir si cette construction est affectée par la présence des bénéfices d'un programme de fidélité. Nous examinons aussi les facteurs de satisfaction, de confiance et d'attachement qui sont considérés comme les principaux contributeurs à la fidélité au programme, et à son tour, à la fidélité à l'enseigne. En plus, l'étude se demande si les différentes formes des bénéfices du programme de fidélité ont un plus grand effet sur la fidélité spécialement dans les grandes et moyennes surfaces et les grandes et moyennes surfaces spécialisées.

Afin de clarifier notre problématique, une étude exploratoire a été menée auprès des enseignes de la grande distribution. Notre étude serve à vérifier les hypothèses et à produire des connaissances permettant de mettre à l'épreuve un cadre théorique prédéfini. Selon Birn (2000), un chercheur ne doit pas nécessairement considérer la recherche quantitative comme une technique autonome, mais plutôt faire usage de techniques qualitatives d'abord afin d'obtenir un sens des attitudes et des opinions des gens, avant qu'une décision finale soit prise de ce qui doit être mesuré. La phase qualitative est composée de deux parties : la première concerne le recueilli de l'information et la méthodologie choisie. La deuxième présente les entrevues personnelles menées avec des représentants de neuf ${ }^{5}$ enseignes qui offrent des programmes de fidélité dans le secteur de la grande distribution. Ces entretiens visent à établir des détails supplémentaires sur les types de programmes qu'ils offrent, ainsi que leurs attitudes et leurs opinions sur les objectifs et les résultats de leurs offres. Ces entretiens, sont utiles pour générer des informations et pour décider de la meilleure façon de mener des recherches supplémentaires. En effet, les réponses des interviewés ont été utilisés pour découvrir des questions qui peuvent être importantes à inclure dans le questionnaire, expliquer comment les répondants pensent, se sentent et parlent du sujet, et aussi pour comprendre comment les clients peuvent réagir à l'égard des offres de récompense. L'information obtenue de notre groupe de discussion va contribuer à faciliter la conception du questionnaire qui va être utilisé dans la phase quantitative de la recherche (Stewart et Shamdasani 2015).

Dans cet article nous aborderons les quatre points suivants : le cadre théorique de la problématique, la méthodologie de l'étude qualitative, les résultats et la discussion de la recherche.

2. Le Cadre théorique : La relation entre les bénéfices des programmes de fidélité et la fidélité à l'enseigne L'objectif majeur des responsables marketing est la fidélité des clients. Selon Reichheld et Schefter, (2000) le principal critère de succès d'une entreprise est d'assurer la fidélité des clients. La fidélité est synonyme de continuité relationnelle, qui permet d'améliorer les performances commerciales d'une enseigne en termes d'augmentation du chiffre d'affaire et de l'achat répète à long terme. L'établissement de relations est donc l'un des principaux moyens pour fidéliser les clients. La force relationnelle est souvent mesurée au moyen de la qualité des relations qui se compose de trois éléments, à savoir la satisfaction, la confiance, et l'attachement (Vesel et Zabkar, 2010). La satisfaction est le résultat du contentement, car les attentes des clients sont satisfaites ou dépassées (Van Vuuren, Roberts-Lombard et van Tonder, 2012). La confiance est un facteur crucial du passage des transactions

\footnotetext{
${ }^{1}$ Ä̈da Lo, "CMI Fidélité : La plateforme au service des commerçants cartonne 》 Publication : 5 mars 2018 ; Affichages : 943. Leseco : http://www.leseco.ma/64168. Consulté le 05/10/2019.

${ }^{2}$ Les enseignes marocaines partenaires dans cette plateforme sont : Sushi Club, Yoka Sushi, Bistrot Burger, Papa John's, Chili’s, La villa Mandarine, Lizarran, Wok to Walk, Le comptoir des Arènes, Le Latium, Eden Island, Wojooh, Sydney Love, Hyatt Place Taghazout, Sofitel Essaouira, Hmizate Go, Coin de Chine, Yatout, Yatout Home, Istikbal, etc.

${ }^{3}$ Le matin du 28 décembre 2018, «Aswak Assalam compte 1 millions d'abonnés à la carte fidélité ». Consulté le 05/10/2019.

${ }^{4}$ Source: The Nielsen Global Retail Loyalty Sentiment survey, 2016.

${ }^{5}$ Nous avons mené 14 entrevues auprès des enseignes de la grande distribution, mais au final nous avons choisi 9 enseignes (critère de choix l'existence des cartes de fidélité).
} 
de marché discrètes à des relations d'échange continues (Dwyer, Schurr et Oh1987), afin de développer et maintenir des relations avec les clients (Morgan et Hunt 1994). L'attachement est décrit comme le produit des comportements qui ont pour objet la recherche et le maintien de la proximité d'une personne (marque et ou enseigne) spécifique (John Bowlby, 1969).

Les programmes de fidélisation est un ensemble de méthodes qui influencent positivement les attitudes et les comportements de fidélité à l'égard du programme, de la marque et de l'entreprise (Henderson, Berk et Palmatier, 2011 ; Meyer-Waarden, 2007 et 2012 ; Sharp et Sharp, 1997). Les enseignes font d'importants investissements dans les programmes de fidélité, mais la question qui se pose est de savoir si cela en vaut la peine. Certains chercheurs affirment que les programmes de fidélité ne sont pas nécessaires (Kreis et Mafael, 2014), car ils considèrent que ces récompenses ne servent pas à enrichir le titulaire de la carte, mais c'est juste un comportement de bienveillance de la part des enseignes pour remercier les clients de leurs achats, qui ne conduit pas nécessairement à la fidélité. En revanche, d'autres chercheurs valident l'importance des bénéfices des programmes de fidélité pour la fidélisation des clients. Ils expliquent que ces bénéfices servent à la fois à modifier et à encourager les comportements d'achat (Kearney, 2013). Les avantages des programmes de fidélité viennent dans différents formats, avec des effets variables sur la qualité de la relation et la fidélité. L'étude s'est concentrée sur trois formes d'avantages qui sont évidents dans les programmes de fidélité. Le premier est le bénéfice utilitaire et probablement le plus courant. Décrit comme économique et monétaire, ce type d'avantage est illustré par des récompenses telles que le cash-back, les remises, les produits gratuits et les prix (Eason, Bing et Smothers, 2015). Les deux autres catégories d'avantages peuvent être considérés comme intangibles, malgré le fait qu'il porte des éléments tangibles qui sont échangés dans le cadre de la transaction. L'une est décrite comme hédoniques, plus personnelle avec des émotions qui en résultent, comme le plaisir du gain de points, traitement préférentiel (Feiler, Tost et Grant, 2012) et les sentiments attachés à des récompenses qui sont données à une autre partie comme une école ou un organisme de bienfaisance (Kareklas, Carlson Muehling, 2014). Alors l'autre est symboliques, plus sociales qui tournent autour de sentiments d'appartenance et d'affiliation.

La relation entre les bénéfices des programmes de fidélité et la fidélité à l'enseigne est le résultat de plusieurs recherches. Principalement, Demoulin et Zidda (2008), qui ont validés que les détenteurs de la carte de fidélité sont considérés comme les clients les plus fidèles à une enseigne et ou marques. Garcia-Gomez et al (2012) ont confirmés que les PF conduit à une fidélité affective envers le magasin. Aussi, Demoulin et Zidda (2009), présentent que les bénéfices en général et symboliques en particulier influencent la fidélité à l'enseigne. MeyerWaarden (2013), indiquent dans son étude que les récompenses augmentent la fidélité. Au final, Ibrahim Idir, 2018, a validé que la fidélité au programme de fidélité est une variable déterminante et intermédiaire entre les bénéfices des PF et la fidélité à l'enseigne.

Toute recherche sur ce sujet doit être à la fois d'intérêt académique et d'intérêt managériale, afin d'apporter des réponses aux enseignes qui s'interrogent encore sur l'utilité des programmes de fidélité. Notre prochaine étape présentera la méthodologie de recherche poursuivie pour établir et analyser les résultats des guides d'entretien.

\section{Méthodologie de l'étude exploratoire.}

L'objectif de l'étude qualitative est d'apporter les éléments de réponse à notre problématique et d'explorer la relation des clients avec les programmes de fidélité dans la grande distribution. En effet, nous cherchons à identifier les leviers de succès des programmes de fidélité des enseignes. Notre méthodologie repose sur un guide d'entretien destiné aux professionnels en charge des programmes de fidélité.

\section{3-1 Analyse de contenu}

L'objectif de l'analyse de données est l'étude des relations des liens entre les variables et les items qui y sont associées dans un texte. Ainsi on peut considérer cette analyse comme un processus d'interprétation des données qui permet d'établir des réponses à des questions. Bardin, (2003) indique que la forme la plus utilisée dans ce contexte est l'analyse de contenu thématique, reposant sur l'identification des thèmes récurrents. La différence entre l'analyse de discours et l'analyse de contenu, est que l'analyse de contenu a pour objectif d'étudier une personne, une parole et extraire des informations d'un corpus sans prendre en considération sa structuration linguistique (Bardin, 2003 ; Berelson, 1952) au contraire de l'analyse de discours. En général, selon Bardin (2007), Miles et Huberman (2003) chaque analyse des données peut se déroulée en trois étapes : la pré-analyse, qui porte sur l'étude et l'organisation des corpus, la saisie des objectifs selon la problématique étudiée et le découpage en unités d'analyse.

\section{3-2 Entretien semi-directif}

L'entretien est l'un des principales méthodes utilisées pour le recueil de données (Romelaer, 2005). Il existe différents types d'entretien selon leur niveau de directivité, principalement l'entretien directif, l'entretien semidirectif et l'entretien non-directif. L'entretien semi-directif est une technique qualitative le plus utilisé sur le terrain. Il est approprié à notre recherche, parce qu'elle porte sur un questionnement précis et permet la comparaison des 
entretiens de plusieurs répondants (Jolibert et Jourdan, 2011). Au niveau de cet entretien on peut aborder plusieurs thématiques sous forme d'une discussion informelle basé sur des réponses spontanées sans contraintes de l'interviewé.

\section{3-3 Guide d'entretien}

La réalisation des entretiens avec les responsables marketing, nécessite en premier lieu une collecte des informations relatives à chaque enseigne à partir des : sites web, brochures et communiqués de la presse en ligne etc. Les responsables marketing interrogés ont été sélectionnés en fonction de leurs expériences et postes dans les enseignes retenus, particulièrement sur la fidélisation des clients. Notre taille de l'échantillon a été déterminée selon le principe de saturation sémantique théorique des entretiens qualitatifs (Glaser et Strauss, 2006). En plus l'échantillon représente les critères qui reflètent les cas possibles de la problématique étudiée (Evrard et al., 2009). En effet, notre critère de sélection retenu est représenté comme suit :

$\checkmark$ Avoir une activité dans la grande distribution ;

$\checkmark$ Avoir des programmes de fidélité ;

Notre guide d'entretien (voir annexe) est mené auprès d'un échantillon de neuf enseignes, sur la base de six thèmes. Ainsi notre échantillon se présente dans le tableau suivant :

Tableau 1 : Présentation des enseignes interviewés

\begin{tabular}{|c|c|}
\hline Sociétés & Villes \\
\hline Aswak Assalam & Mohammedia / Kenitra \\
\hline Marjane & Casablanca / Salé \\
\hline Market plus & Casablanca \\
\hline Atacadao & Salé / Rabat \\
\hline Ikea & Casablanca \\
\hline Mr Bricolage & Casablanca \\
\hline Brico déco & Casablanca \\
\hline Virgin Mégastore & Rabat \\
\hline Bricoma & Salé / Kenitra \\
\hline
\end{tabular}

Source : Propre conception de l'auteur

\section{3-4 Analyse des données à l'aide du logiciel « NVivo »}

La retranscription a été établie sur le logiciel « NVivo » afin de porter une analyse de contenu thématique appliquer par des unités de découpage, de codage et, ensuite, d'analyse (Evrard et al., 2009). Selon Berelson (1952) (cité dans Evrard et al., 2009) explique que c'est « une technique de recherche pour la description objective, systématique, et quantitative du contenu manifeste des communications ayant pour but de les interpréter ». Ainsi notre analyse exploratoire nous a permis de remplir la fonction «comprendre » (Bardin, 2007). L'analyse lexicale présente la réduction du volume d'informations à analyser, plus particulièrement les corpus de grande taille. Le logiciel «NVivo» offre par ailleurs la possibilité d'établir des tableaux, graphes, etc permettant de faciliter la lecture, l'interprétation des discours et la classification des résultats par ordre de fréquence. Les résultats proposés par le logiciel sont adaptés aux objectifs des études exploratoires de notre recherche.

Sur la base de la méthodologie de collecte et d'analyse des données, nous allons présenter, dans la prochaine étape les résultats obtenus.

\section{Les résultats de l'étude qualitative.}

Les résultats obtenus à partir de nos guides d'entretiens ont montré que la plupart des répondants souhaitent une récompense immédiate et tangible (bénéfice utilitaire), particulièrement dans les grandes et moyennes surfaces. En effet, les clients préfèrent d'associer l'achat avec l'avantage. Les interviewés ont expliqué que les adhérents apprécient d'être reconnus pour leurs habitudes d'achat, ainsi que de profiter des récompenses monétaires. Toutefois, cela ne s'est pas nécessairement traduit par la fidélité, car les membres peuvent faire le choix d'un magasin selon les avantages présentés par ces enseignes. La fidélité ne semble pas être une conclusion lorsque les consommateurs possèdent une carte de fidélité, malgré l'expérience positive de recevoir une récompense. Par contre, la fidélité est le résultat de la satisfaction, la confiance et l'attachement (variable qui ressorte de notre étude qualitative) des clients dans les bénéfices du programme de fidélité par l'intermédiaire de la fidélité au programme. La fidélité envers une enseigne peut être décrite, lorsque le client fréquente régulièrement le magasin et fait des recommandations positive à d'autres clients (Cant et du Toit, 2012).

Les principaux résultats de notre recherche qualitative sont présentés dans les points suivants :

\section{4-1 Construction de la fidélité au programme}

Les dimensions utilitaires, hédonique et symboliques sont des déterminants important de la fidélité au programme 
(par l'intermédiaire de la satisfaction, la confiance et l'attachement). Ces dimensions sont bien présentent dans le verbatim des interviewés qui évoquent que les adhérents ont des émotions de satisfaction, de joie ainsi que d'autres sentiments ludiques et sociales liés au traitement préférentiel, des invitations à des événements spéciaux, le sentiment d'appartenance et de reconnaissance etc.

La construction de la fidélité au programme à partir de notre étude qualitative exploratoire et de la littérature, nous a permis de présenter les nouveautés introduites dans notre modèle conceptuel suivantes :

$>$ Introduction de l'attachement en tant que déterminant de la variable fidélité au programme et fidélité à l'enseigne.

> Proposition d'annulation de l'hypothèse entre les bénéfices des PF et de la fidélité au programme (s'il ne y'a pas introduction des variables satisfaction, confiance et attachement) ;

Ainsi nous avons enrichi notre modèle de recherche par les thèmes suivants :

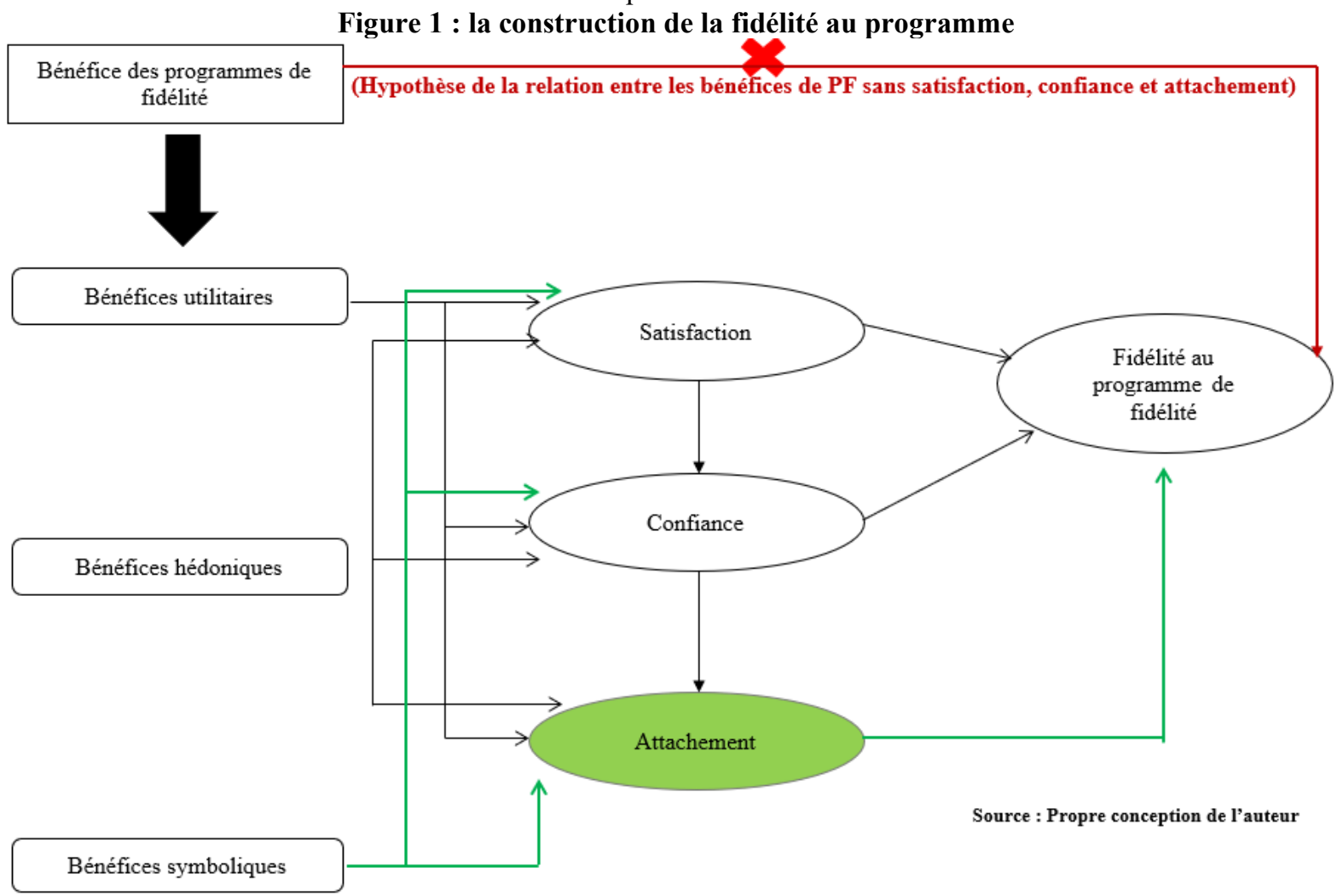

\section{4-2 Construction de la fidélité à l'enseigne}

Les déterminants de la fidélité à l'enseigne sont présentés par les variables suivantes :

$>$ La satisfaction : La satisfaction à l'égard des PF reflète l'état affectif du client résultant d'une évaluation globale des avantages reçus du PF (Oliver, 1997). La satisfaction des clients dans les bénéfices des programmes de fidélité est une variable très importante expliquant la fidélité des clients au programme et à l'enseigne. En effet, des recherches par exemple, Mimouni-Chaabane et Volle, (2010) ; Yi et Jeon, (2003) suggèrent que les clients sont plus satisfaits de leur PF lorsqu'ils perçoivent ses avantages comme importants.

La confiance : Il existe plusieurs travaux dans la littérature marketing qui ont validés l'influence positive de la confiance sur la fidélité des clients exemple : Chaudhuri et Holbrook, (2001); Sirdeshmukh, Singh et Sabol, (2002); Aurier et al., (2001/2010); Roostika, (2011). C'est une variable d'ordre cognitif présentée dans notre étude à travers deux dimensions « l'intégrité » et « la bienveillance ». Selon Morgan et Hunt, 1994_la confiance renforce l'échange social en se basant sur « la fiabilité » et «l'intégrité » entre le client et l'enseigne.

$>$ L'attachement : c'est une variable construit à travers plusieurs états psychologiques. Dans ce même sens, il traduit une relation affective entre le client et la marque / enseigne (Lacoeuilhe, 2000 et 2007). Cette variable prend la dernière place dans la chaine relationnelle de fidélité selon Aurier et al., (2001) et (2007).

$>$ La fidélité au programme : Selon Evanschitzky et al. (2012) la fidélité au programme est une fidélité basée sur les bénéfices économiques. C'est une fidélité par laquelle la valeur perçue des bénéfices est un prédicteur majeur de la fidélité au programme. 
En général, notre analyse confirme que la satisfaction renforce la confiance qu'est un déterminant de l'attachement. Ces trois variables conduit à la fidélité au programme et par la suite à la fidélité à l'enseigne. La construction de la fidélité à l'enseigne à partir des verbatim et de la littérature, nous a permis de présenter le modèle suivant :

Figure 2 : la construction de la fidélité à l'enseigne

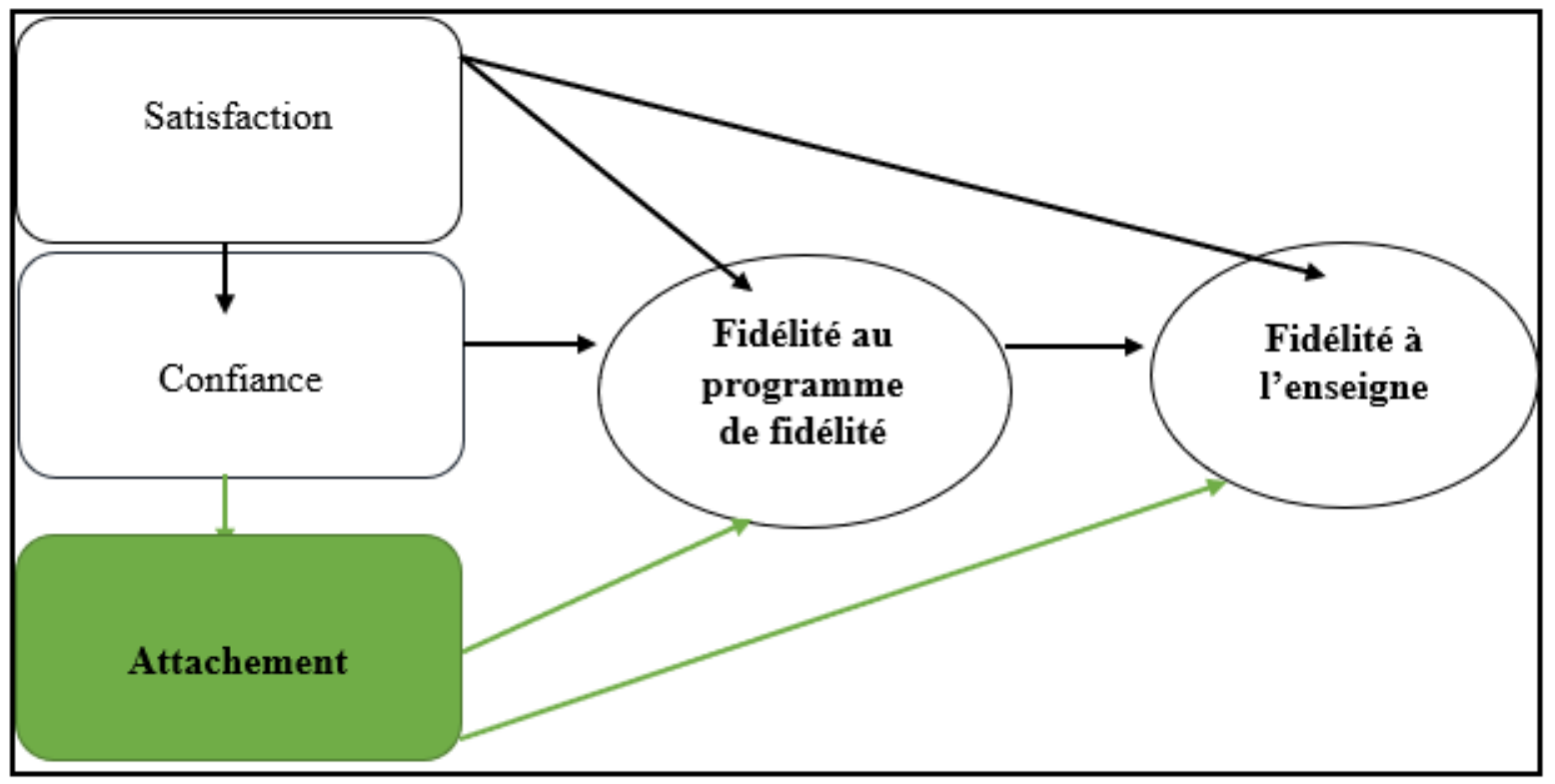

Source : Propre conception de l'auteur

\section{4-3 Conséquences de la fidélité}

À partir de notre étude qualitative et de la littérature marketing, nous avons pu enrichir notre modèle conceptuel par les nouvelles variables (les conséquences de la fidélité à l'enseigne) suivantes :

$>$ Le bouche-à-oreille ${ }^{1}$ positif : Selon Dick et Basu, (1994) les clients peuvent exprimer leur satisfaction/insatisfaction à leur entourage en conséquence de l'utilisation d'un service/produit (dans notre étude c'est l'expérience avec une enseigne), c'est ce qu'on appelle le bouche-à-oreille. Cette dernière peut être positive ${ }^{2}$ ou négative ${ }^{3}$ (Reichheld, 2003).

$>\quad$ L'intention à la fidélité : c'est la réponse positive à la satisfaction des clients. Selon les responsables des enseignes cette intention est considérée comme une conséquence de la fidélité à l'enseigne.

> La résistance à la contre persuasion : c'est un processus associé à l'attitude par lequel les clients peuvent rester fidèles malgré les opportunités auprès des autres concurrents. Selon N'Goala (2010) la fidélité est considérée comme l'expression de résistance à l'opportunisme.

$>\quad$ La part du portefeuille client ${ }^{4}$ : cette part est représentée dans notre étude par le nombre des adhérents de la carte de fidélité des enseignes, qui ont un statut toujours active.

$>$ L'achat répété : l'un des principales conséquences de la fidélité à l'enseigne est l'achat répété. La répétition de l'achat représente la satisfaction, la confiance et même l'attachement des adhérents dans les bénéfices des PF de l'enseigne. Jean Frisou (2006) explique la théorie de l'achat répété la fidélité par la répétition du comportement d'achat. Si les adhérents à une carte de fidélité obtiennent une récompense, il aura une plus forte probabilité de répéter l'achat.

\section{4-4 Présentation des résultats selon le logiciel Nvivo}

Les résultats du logiciel Nvivo seront présentés par les figures suivantes : le nuage des mots, diagramme hiérarchique des nœuds et le dendrogramme des nœuds parents par similarité de mots.

\footnotetext{
${ }^{1}$ Word-of-mouth « WOM ».

${ }^{2}$ Clients promoteurs.

${ }^{3}$ Clients détracteurs.

${ }^{4}$ Share-of-Wallet (SOW) : dans notre étude on prend des adhérents toujours actif.
} 


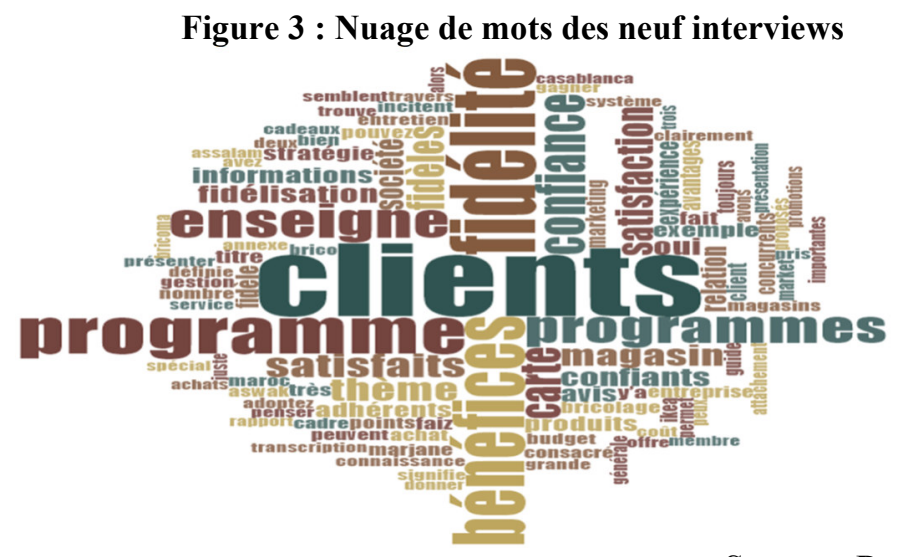

Source : Propre conception de l'auteur

La présentation des nuages de mots indique que le terme client prend le lead dans la globalité des transcrites analysée, suivit, par la fidélité et les bénéfices.

Figure 4 : Diagramme hiérarchique des nœuds

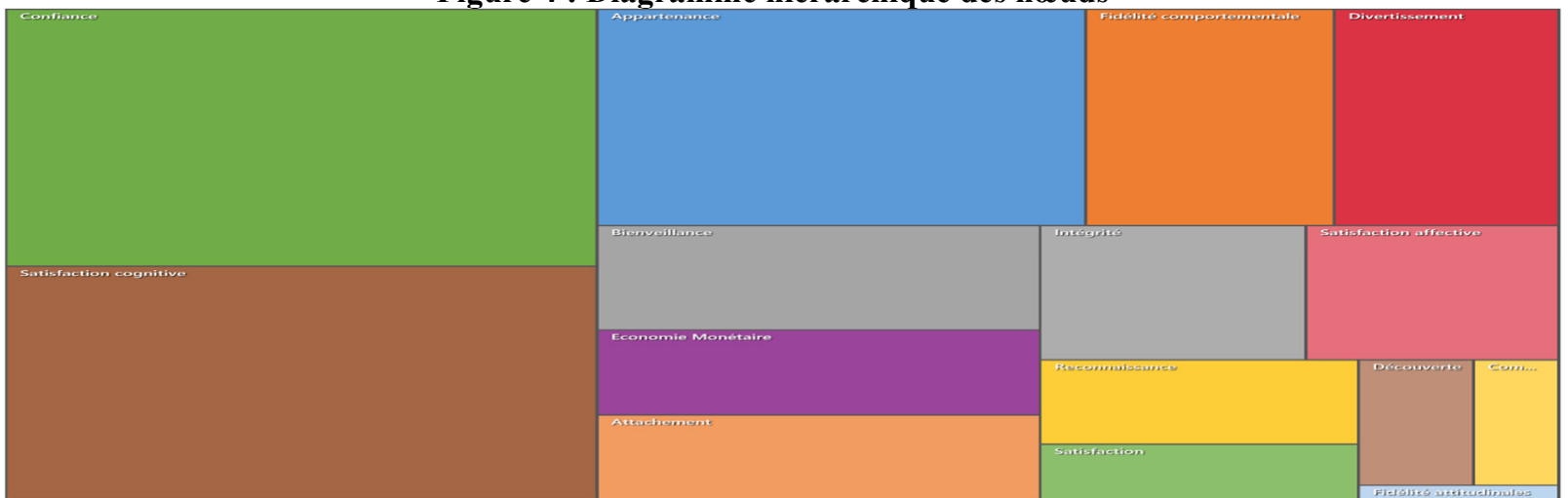

Source : Propre conception de l'auteur

Selon ce diagramme hiérarchique, on trouve la confiance prend la première position suivie par la satisfaction cognitive pour arriver au final à la fidélité attitudinale.

Figure 6 : Dendrogramme des nouds parents par similarité de mots

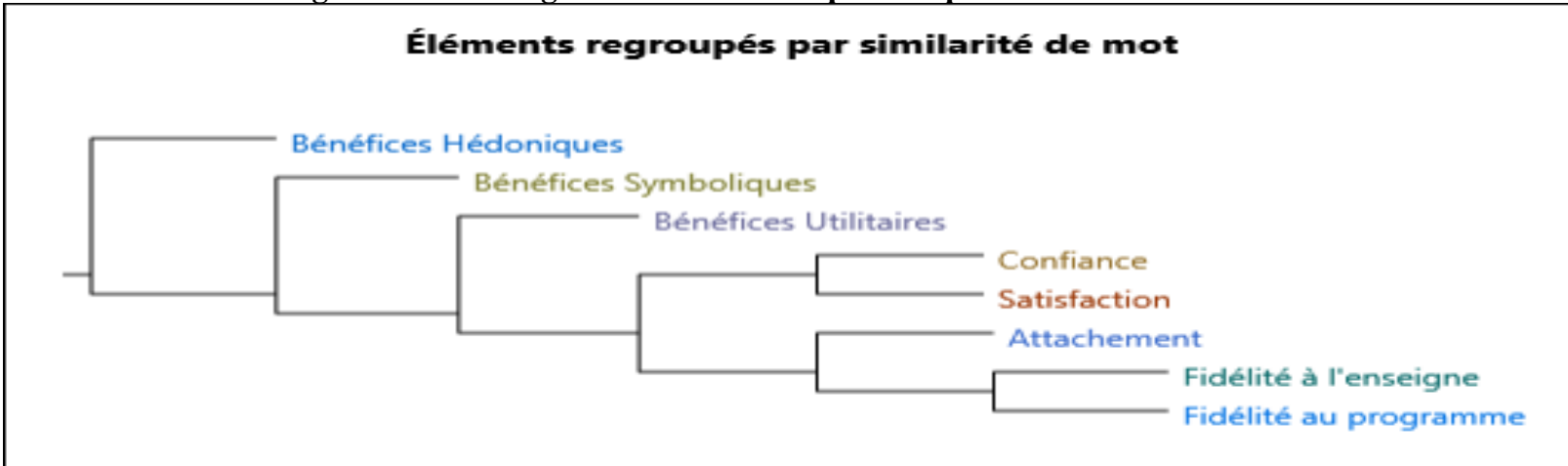

Source : Propre conception de l'auteur

A partir de l'analyse du dendrogramme on trouve que la fidélité au programme et la fidélité à l'enseigne sont les nœuds résultants. L'organisation du dendrogramme des similarités de mots faite sur la base du coefficient de corrélation Pearson ${ }^{1}$, nous donne un schéma semblable à celui de notre modèle conceptuel.

\section{4-5 Présentation du modèle conceptuel final}

L'étude des résultats obtenu à partir du logiciel Nvivo et l'analyse de contenu nous a permis de présenter notre model conceptuel final suivant :

\footnotetext{
${ }^{1}$ Les coefficients de corrélation permettent de donner une mesure synthétique de l'intensité de la relation entre deux caractères et de son sens lorsque cette relation est monotone. Le coefficient de corrélation de Pearson permet d'analyser les relations linéaires et le coefficient de corrélation de Spearman les relations non-linéaires monotones. Il existe d'autres coefficients pour les relations non-linéaires et non-monotones.
} 
Figure 7 : Modèle Conceptuel détaillé (avec hypothèses)

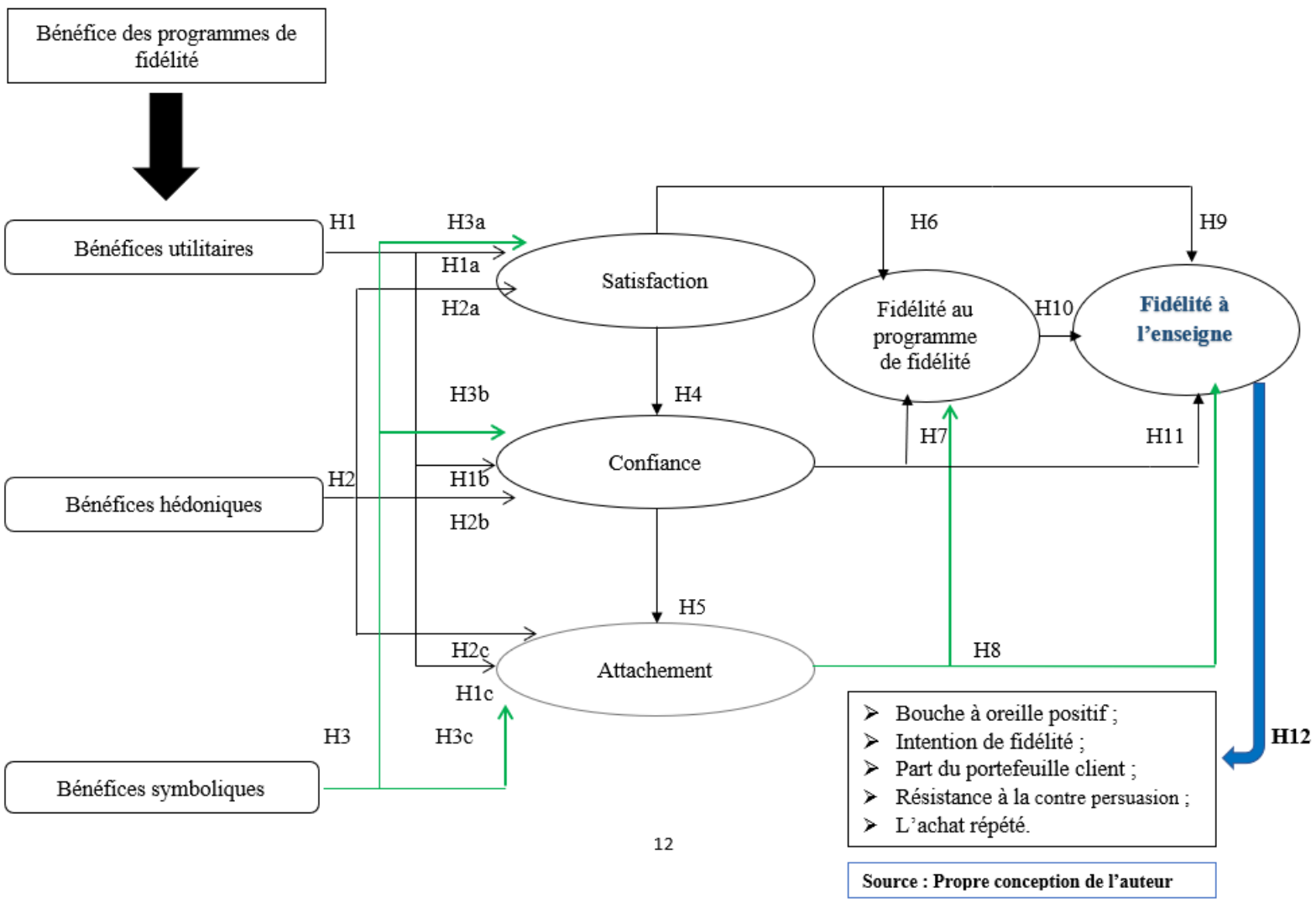

\section{Discussion}

L'étude qualitative exploratoire nous a permis d'enrichir notre modèle conceptuel par l'apparition de nouvelles variables. Notamment, l'attachement et les conséquences de la fidélité : le bouche à oreille positif, la part du portefeuille client, la résistance à la contre persuasion, l'intention de fidélité et l'achat répété.

L'attachement est une variable qui s'exprime par un sentiment de proximité entre le consommateur et la marque (et/ou enseigne) (Lacoeuilhe, 2000a). Il est un lien émotionnel et affectif à la marque (et/ou l'enseigne) (Lacoeuille, 2000b ; Fournier, 1998 ; Aurier et al., 2001). De même, J. Lacoeuille, (1997) a indiqué que l'attachement est un facteur expliquant la fidélité.

Concernant les conséquences de la fidélité, de nombreuses études ont expliqué ses composantes : l'achat répété est considéré comme un comportement qui conduit à la fidélité. Selon Noble et Phillips, (2004) les programmes de fidélité sont couramment utilisés par les détaillants pour renforcer les relations avec les clients et encourager le patronage en les récompensant avec des bénéfices pour chaque comportement répété. Pour la part de portefeuille, Omar et al., $(2011 \mathrm{~b})$ comme d'autres chercheurs ont validés la relation positive entre la fidélité à l'enseigne et l'évolution de la part du portefeuille client dû aux bénéfices des programmes de fidélité. Gâmez, Arranz et Cillon (2006) ont indiqué que la fidélité a une influence positive sur la propension du consommateur à émettre un bouche-à-oreille positif. On ajoute aussi, que de nombreuses études ont examiné la relation positive entre la satisfaction et l'intention de rester fidèle à l'enseigne et/ou marque (Zeithaml et al., 1996 ; Leenheer et al, 2007). Au final, la résistance à la contre persuasion, c'est le fait de rester fidèle malgré les opportunités des concurrents. Selon N'Goala (2010) la fidélité est l'expression de la résistance à l'opportunisme.

L'analyse des données de la présente étude nous a permis de faire sortir d'autres résultats. Notamment, l'existence de plusieurs facettes de la fidélité : les «faux fidèles» et les «multi-fidèles» où le client peut avoir en même temps, plusieurs carte de fidélité et recherche des bénéfices auprès un grand nombre d'enseignes. Ces clients sont opportunistes, appelés aussi «chasseurs de promotions» (Meyer-Waarden, 2002).

On ajoute que, les bénéfices utilitaires sont les plus préférés par les clients dans le secteur de la grande distribution sauf le cas des produits chers (de luxe) où le client reste exigeant au niveau de la qualité de service et le traitement préférentiel (bénéfices hédoniques et symboliques).

Nous avons trouvé aussi, qu'il existe un unique système du programme de fidélité, qui est la carte avec gestion de point présentée par chaque enseigne. Cependant, on propose de créer une carte de fidélité aux clients qui permet aux utilisateurs de bénéficier des avantages avec plusieurs partenaires dans plusieurs secteurs (automobile, 
tourisme, la grande distribution, le transport, etc).

De même nous avons remarqué que, malgré la popularité des PF, certains clients subsistent encore des lacunes dans la compréhension des avantages de ces programmes (Meyer-Waarden, Benavent et Castéran, 2013). Les responsables interviewés indiquent, qu'un certain nombre de clients ont des difficultés pour comprendre le fonctionnement du programme, d'échanger les récompenses et d'avoir les informations fiables sur ce qui a été réellement à leur disposition en tant que membres du programme. Pour cette raison, la satisfaction, la confiance et l'attachement, des clients aux bénéfices varient selon la compréhension des types de programmes. Certains répondants indiquent que les clients sont satisfaits de leurs programmes, il s'agit principalement des programmes où le titulaire de la carte été bien informé et peut clairement lier les achats à leur récompense. En revanche, la satisfaction été moins évidente lorsque les programmes ${ }^{1}$ ont été difficiles à comprendre par les clients, dû au manque d'explication et de publicité. L'élément de confiance a été lié aux mêmes questions. Les clients se sentent plus confiants lorsque la communication fait clairement partie du programme. Les titulaires de carte ayant le sentiment d'avoir été trompés s'ils n'ont pas pu profiter de leurs bénéfices due à la complication et manque d'explication du programme. L'attachement a été accru lorsque les titulaires de carte ont estimé que les avantages du programme couvrent une partie des coûts engagés. A cet effet, nous proposons aux enseignes de présenter un programme efficace, simple, clair avec une stratégie de communication et de publicité pertinente et ciblée.

Finalement, nous constatons que notre échantillon interviewé a validé la relation positive entre les bénéfices des PF et la fidélité à l'enseigne en passant par les variables satisfaction, confiance, attachement et fidélité au programme.

\section{Conclusion}

Les programmes de fidélité sont en plein renouveau. La tendance aujourd'hui, se concentre sur l'intérêt des clients, particulièrement les adhérents aux cartes de fidélités. Les titulaires de cartes sont des clients diversifiés, mieux informés et conscients de sa valeur. D'ailleurs, ils sont davantage à la recherche de sensations et de découvertes. Ils veulent se sentir valorisés et privilégiés, bref, ils souhaitent que les enseignes les comprennent et les trouvent importants. En effet, conserver le client existant dans ce monde concurrentiel est une tâche difficile. Il exige d'immenses efforts, diverses stratégies et des programmes innovants de fidélisation. Pour cette raison, ces dernières années, les programmes de fidélisation de la clientèle ont radicalement changé. Les nouveaux programmes sont devenus numériques, efficaces et uniques afin de rendre le client heureux et d'augmenter son engagement.

Notre recherche qualitative est un précurseur à une étude quantitative plus pertinente. Les résultats obtenus à partir de l'étude des guides d'entretien nous ont permis de confirmer ou d'infirmer, nos hypothèses et variables de recherche. En plus, elle va nous servir à bien détailler le choix des thèmes du questionnaire. Ces résultats ont montré que les bénéfices des programmes de fidélité sont essentiels pour établir des relations de fidélité à long terme. En outre, les programmes doivent être simples et clairement définis afin de promouvoir la satisfaction, la confiance et l'attachement. L'étude a révélé aussi que, les conséquences comportementales et attitudinales de la fidélité sont présentés par : l'achat répété, le bouche-à-oreille positif, la résistance à la contre persuasion, l'intention de fidélité et la part du portefeuille client.

En général, les enseignes offrent des récompenses aux clients en échange des coûts encourus, afin d'atteindre un seul objectif qu'est la fidélité à l'enseigne. Par la suite, cette étude devrait aider les enseignes à isoler les types de récompenses qui ont un faible effet sur la fidélité des clients.

La recherche sur ce sujet est à la fois d'intérêt académique et pratique, fournissant des réponses aux magasins qui peuvent remettre en question l'utilité des programmes de fidélité et / ou en considérant les types de programmes les plus susceptibles de contribuer à la fidélité des clients. Elle permettra aussi, aux enseignes nationales de créer des offres qui leur permettent de se différencier de leurs concurrents. Les programmes de fidélisation sont coûteux à lancer et à exécuter, et il est donc nécessaire de se demander si les entreprises versent en vain de grosses sommes d'argent ou si les programmes augmentent réellement leurs revenus. Elle permet aussi de catégoriser les avantages perçus par les programmes de fidélité en trois domaines, à savoir utilitaires, hédoniques et symboliques (tels que : gain des points, promotions, cadeaux, récompenses sociaux etc) et s'interroge sur la façon dont chaque forme contribue à la fidélité au programme et à l'enseigne.

Les limites de cette étude qualitative exploratoire sont présentées par les deux points suivants : premièrement, notre terrain de recherche a plusieurs contraintes administratives, que ça soit dans la prise de rendez-vous, ou dans les réponses aux questions tel que le budget consacré au PF et chiffre d'affaire actualisé. Deuxièmement, les résultats de notre recherche se limitent seulement à un seul secteur qu'est la grande distribution et un seul territoire qu'est le marché marocain, cela peut conduire au développement futur de notre recherche dans d'autres cultures.

$\mathrm{Au}$ final, nous proposons de mener et prolonger notre recherche par l'étude des théories et modèles sur la motivation des clients et l'innovation dans les bénéfices des programmes de fidélité. De même, on propose d'élargir cette étude vers le marché de télécommunication particulièrement l'opérateur télécom Inwi qui est la plus

\footnotetext{
${ }^{1}$ Les bénéfices du programme de fidélité n'été pas claire ni bien expliqué.
} 
avant-gardiste société en matière de bénéfice du programme de fidélité à travers le « club Inwi ».

\section{References}

Aurier Ph. Et Fort F. (2007), The effects of perceived congruity between origin, brand and product on the purchase intention of a branded product of origin, Competitive Paper, Advances in Consumer Research, vol 34.

Aurier Ph. et Siadou Béatrice (2007), Perceived Justice and Consumption Experience Evaluations: A Qualitative and Experimental Investigation, International Journal of Service Industry Management, 18, 5, 450-471.

Aurier, P. and de Lanauze, G.S. 2012. Impacts of perceived brand relationship orientation on attitudinal loyalty: An application to strong brands in the packaged goods sector. European Journal of Marketing, 46(11/12): $1602-1627$.

Aurier, P., Benavent, C., et N'Goala, G. (2001). Validité discriminante et prédictive des composantes de la relation à la marque. 17éme Actes du congrès de l'AFM, 156-159.

Aurier, P., et N'Goala, G. (2010). The differing and mediating roles of trust and relationship commitment in service relationship maintenance and development. Journal of the Academy of Marketing Science, 38, 3, 303-325.

Bardin, L. (2003), L’Analyse de contenu, Paris : Presses Universitaires de France (PUF), 10 ème édition.

Bardin, L. (2007), L’Analyse de contenu, Paris : Presses Universitaires de France (PUF).

Berelson, B. (1952), Content Analysis in Communication Research, Glencoe: The Free Press.

Birn, R. J. 2000. The handbook of international market research techniques. 2nd ed. London: Kogan Page.

Bojei, J., Julian, C. C., Wel, C. A. B. C. and Ahmed, Z. U. (2013). The empirical link between relationship marketing tools and consumer retention in retail marketing. Journal of Consumer Behaviour, 12(3): 171-181.

Bowlby, J. (1969). Attachement et perte, vol.1:L'attachement. Paris: PUF. Jolibert, A. et Jourdan, P. (2011), Marketing research. Méthodes de recherche et d'études en marketing, Paris : Dunod. Journal of Sociology, 50(4), 279-283.

Cant, M. C. and du Toit, M. N. (2012). Identifying the factors that influence retail customer loyalty and capitalising them. International Business and Economics Research Journal, 11(11): 1223-1232.

Chaudhuri, A., et Holbrook, M. (2001). The chain of effects from brand trust and brand affect to brand performance the role of brand loyalty. Journal of Marketing, 65, 2, 81-93.

Demoulin N., Zidda P., (2009), Drivers of Customers' Adoption and Adoption Timing of a New Loyalty Card in the GroceryRetail Market, Journal of Retailing, 85(3), pp. 391-405.

Demoulin, N., et Zidda, P. (2008). On the impact of loyalty cards on store loyalty: Does the customers' satisfaction with the reward scheme matter? Journal of Retailing and Consumer Services, 15, 5, 386-398.

Dick, A.S., et Basu, K. (1994). Customer loyalty: Toward an integrated conceptual framework. Journal of the Academy of Marketing Science, 22, 2, 99-113.

Dwyer, F.R., Schurr, P.H., et Oh, S. (1987). Developing buyer-seller relationships. Journal of Marketing, 51, 2 , 11-27.

Eason, C. C., Bing, M. N. and Smothers, J. 2015. Reward me, charity, or both? The impact of fees and benefits in loyalty programs. Journal of Retailing and Consumer Services, 25: 71-80.

Evanschitzky, H., Ramaseshan, B., Woisetschläger, D.M., Richelsen, V., Blut, M., et Backhaus, C. (2012). Consequences of customer loyalty to the loyalty program and to the company. Journal of the Academy of Marketing Science, 40, 5, 625-638.

Evrard, Y., Pras, B. et Roux, E. (2009), Market : Etudes et Recherches en Marketing, Paris : Dunod, 4ème édition.

Evrard, Y., Pras, B., Roux, E., Desmet, P., Dussaix, A.M., et Lilien, G.L. (2009). Market-fondements et méthodes des recherches en marketing. Dunod. Paris. Exchanges. Journal of Marketing, 66, 1, 15-37.

Feiler, D. C., Tost, L. P. and Grant, A. M. 2012. Mixed reasons, mixed giving: the costs of blending egoistic and altruistic reasons in donation requests. Journal of Experimental Social Psychology, 48: 1322-1328. .

Fournier, S. (1998). Consumers and their brands: Developing relationship theory in consumer research. Journal of Consumer Research, 24, 4, 343-353.

García-Gómez, B., Gutiérrez-Arranz, A. and Gutiérrez-Cillán, J. 2006. The role of loyalty programs in behavioural and affective loyalty. Journal of Consumer Marketing, 23(7): 387-396.

García-Gómez, B., Gutiérrez-Arranz, A. and Gutiérrez-Cillán, J. 2012a. Drivers of customer likelihood to join grocery retail loyalty programs: an analysis of reward programs and loyalty cards. Journal of Retailing and Consumer Services, 19: 292-500.

García-Gómez, B., Gutiérrez-Arranz, A. and Gutiérrez-Cillán, J. 2012b. Exploring the influence of three types of grocery retailer loyalty programs on customer effective loyalty. The International Review of Retail, Distribution and Consumer Research, 22(5): 547-561.

Glaser et Strauss, 2006: Référence citée dans l'ouvrage : Evrard, Y., Pras, B., Roux, E., Desmet, P., Dussaix, A.M., et Lilien, G.L. (2009). Market-fondements et méthodes des recherches en marketing. Dunod. Paris.

Henderson, C.M., Beck, J.T., et Palmatier, R.W. (2011). Review of the theoretical underpinnings of loyalty programs. Journal of Consumer Psychology, 21, 3, 256-276. 
Idir, B. (2018). L'impact des bénéfices perçus des programmes de fidélisation sur la fidélité à l'enseigne : le rôle médiateur de la fidélité au programme de fidélité. CEPN Working Papers 2018-03, Centre d'Economie de l'Université de Paris Nord.

Jean, Frisou. (2006). Fidélité et vérité : une question philosophique aux enjeux théoriques et managériaux. Cairn.info. 2010/1 n 31 | pages 287 à 303. ISSN 1768-5958.

Kareklas, I., Carlson, J. R. and Muehling, D. D. 2014. "I eat organic for my benefit and yours": egoistic and altruistic considerations for purchasing organic food and their implications for advertising strategists. Journal of Advertising, 43(1): 18-32.

Kearney, 2013. It is raining rewards. Personal Finance. 2013. July 23. Available: http: //www.iol.co.za/business/personal-finance/it-s-raining-rewards-1551488.VLY95CuUc3k. (Accessed 4 June 2014).

Kreis, H. and Mafael, A. (2014). The influence of customer loyalty program design on the relationship between customer motives and value perception. Journal of Retailing and Consumer Services, 21(4): 590-600.

Lacoeuilhe J. (1997), Le rôle du concept d'attachement dans la formation du comportement de fidélité, Revue Française de Marketing, 165, 5, pp. 29-42.

Lacoeuilhe, J. (2000). L'attachement à la marque : proposition d'une échelle de mesure. Recherche et Applications en Marketing, 15, 4, 61-77.

Lacoeuilhe, J., et Belaïd, S. (2007). Quelle (s) mesure (s) pour l'attachement à la marque ? Revue Française du Marketing, 213, 7-25.

Leenheer, J., Van Heerde, H.J., Bijmolt, T.H.A, et Smidts, A. (2007). Do loyalty programs really enhance behavioral loyalty? An empirical analysis accounting for self-selecting members. International Journal of Research in Marketing, 24, 1, 31-47.

Melnyk, Valentyna; Bijmolt, Tammo, (2015). The effects of introducing and terminating loyalty programs. In: European Journal of Marketing. Vol. 49, No. 3-4. pp. 398-419.

Meyer-Waarden L. (2016), The effects of loyalty programs rewards' timing, tangibility and image compatibility on value perception and store loyalty, European Marketing Academy Conference, Oslo.

Meyer-Waarden, L. (2002). Les sources d'efficacité des programmes de fidélisation : une étude empirique sur la base d'un panel single source. Thèse pour le doctorat en sciences de gestion. Université de Pau.

Meyer-Waarden, L. (2007). The effects of loyalty programs on customer lifetime duration and share of wallet. Journal of Retailing, 83, 2, 223-236.

Meyer-Waarden, L. (2012). Management de la fidélisation. Vuibert éditeur. Paris.

Meyer-Waarden, L. (2013). The impact of reward personalization on frequent flyer programmers' perceived value and loyalty. Journal of Services Marketing, 27, 3, 183-194.

Meyer-Waarden, L., Benavent, C. and Castéran, H. 2013. The effects of purchase orientations on perceived loyalty programmes' benefits and loyalty. International Journal of Retail \& Distribution Management, 41(3): 201225.

Miles, M.B., et Huberman, A.M. (2003). Analyse des données qualitatives. (Eds) De Boeck Supérieur. Bruxelles.

Mimouni-Chaabane, A. et Volle, P. (2010) Perceived benefits of loyalty programs: Scale development and implications for relational strategies. Journal of Business Research, 63(1): 32-37.

Morgan, R.M., et Hunt, S.D. (1994). The commitment-trust theory of relationship marketing. Journal of Marketing, 58, 3, 20-38.

N'Goala, G. (2000). Une approche fonctionnelle de la relation à la marque : de la valeur perçue des produits à la fidélité des consommateurs. Thèse pour le doctorat en sciences de gestion. Université de Montpelier.

Oliver, R.L. (1997). Satisfaction: a behavioral perspective on the consumer. New York: McGraw-Hill.

Oliver, R.L. (1997). Sustainable competitive advantage: Combining institutional and resource based views. Strategic Management Journal, 18, 9, 697-713.

Omar, N. A., Ramly, S. M., Syed Shah Alam, S. S. A., et Nazri, M. A. (2015). Assessing the effect of loyalty program benefits in satisfaction- loyalty relationship: Evidence from Malaysia. Jurnal Pengurusan, 43, 145159.

Omar, N.A., Alam, S.S., Aziz, N.A. et Nazri, M.A. (2011) a. Retail loyalty programs in Malaysia: The relationship of equity, value, satisfaction, trust, and loyalty among cardholders. Journal of Business Economics and Management, 12(2): 332-352.

Omar, N.A., Aziz, N.A. et Nazri, M.A. (2011) b. Understanding the relationships of program satisfaction, program loyalty and store loyalty among cardholders of loyalty programs. Asian Academy of Management Journal, 16(1): 21-41.

Reichheld, F.F. and Schefter, P. (2000) E-Loyalty: Your Secret Weapon on the Web. Harvard Business Review, 78, 105-113. Reichheld, 2003.

Romelaer, P. (2005), L'entretien de recherche. In P. Roussel \& F. Wacheux F. (Eds.), Management et ressources humaines. Méthodes de recherche en sciences sociales, Bruxelles : De Boeck University. 
Roostika, R. (2011). The effect of perceived service quality and trust on loyalty: Customer's perspectives on mobile internet adoption. International Journal of Innovation, Management and Technology, 2, 4, $286-291$.

Sharp, B., et Sharp, A. (1997). Loyalty programs and their impact on repeat-purchase loyalty patterns. International Journal of Research in Marketing, 14, 5, 473-486.

Sirdeshmukh, D., Singh, J., et Sabol, B. (2002). Consumer trust, value, and loyalty in relational.

SM Noble, J Phillips Relationship hindrance: why would consumers not want a relationship with a retailer? Journal of Retailing 80 (4), 289-303.

Stewart, D. W. and Shamdasani, P. N. 2015. Focus Groups: theory and practice. 3rd ed. Thousand Oaks, CA: Sage.

The Nielsen Global Retail Loyalty Sentiment survey, 2016.

Van Vuuren, T., Roberts-Lombard, M. and van Tonder, E. 2012. Customer satisfaction, trust and commitment as predictors of customer loyalty within an optometric practice environment. South African Business Review, 16(3): 81-96.

Vesel, P. and Zabkar, V. 2010a. Comprehension of relationship quality in the retail environment. Managing Service Quality, 20(3): 213-235.

Vesel, P. and Zabkar, V. 2010b. Relationship quality evaluation in retailers' relationships with consumers. European Journal of Marketing, 44(9/10): 1334-1365.

Yi, Y., et Jeon, H. (2003). Effects of loyalty programs on value perception, program loyalty, and brand loyalty. Journal of the Academy of Marketing Science, 31, 3, 229-240.

Zeithaml, V.A., Berry, L.L. et Parasuraman, A. (1996), The Behavioral Consequences of Service Quality, Journal of Marketing, 60(2), 31-46.

\section{Thème 1 : Présentation générale de la société (Magasin) \\ 1- Pouvez-vous nous présenter votre société (Magasin)? \\ 2- Quels sont vos clients, produits et concurrents?}

Thème 2 : Le système du programme de fidélisation de la société (Magasin)

1- Quels sont les programmes de fidélisation que vous adoptez ? Quels sont les bénéfices de ces programmes ?

2- Quel est le budget et le coût de gestion consacré pour les programmes de fidélisation?

3- L'entreprise a-t-elle une stratégie marketing clairement définie dans le cadre des PF ? Comment ?

Thème 3 : Les bénéfices du programme de fidélité et la satisfaction.

1- D’après votre expérience, est-ce que les clients sont satisfaits des bénéfices du PF ? A quel titre ?

2- A votre avis comment avez-vous pris connaissance de ces informations ?

3- Est ce que les clients satisfaits sont les plus confiants au PF ?

Thème 4 : Les bénéfices du programme de fidélité et la confiance.

1- D'après votre expérience, est-ce que les clients ont confiance dans les bénéfices du PF ? A quel titre ?

2- A votre avis qu'est ce qu'il vous fait penser que les clients ont confiance aux programmes ?

Thème 5 : Les bénéfices du programme de fidélité et la fidélité au programme.

1- Est-ce que les bénéfices proposés par votre société (magasin) incitent les clients à être fidèle aux programmes?

2- A votre avis est-ce que les clients qui sont satisfaits des bénéfices sont les plus fidèles aux programmes?

3- Est-ce les clients confiants aux bénéfices sont les plus fidèle aux programmes?

Thème 6 : Les bénéfices du programme de fidélité et la fidélité à l'enseigne.

1- Est-ce que les clients satisfaits sont les plus fidèles à l'enseigne ? Comment ?

2- Est-ce que les clients confiants aux bénéfices sont les plus fidèles à l'enseigne ? Comment?

3- Ya-t-il une relation entre fidélité au programme et fidélité à l'enseigne ? Pourquoi ?

4- Est-ce qu'il y a d'autres informations qui vous semblent importantes ? 